\title{
Publisher Correction: Nanoplasmonic electron acceleration by attosecond-controlled forward rescattering in silver clusters
}

Johannes Passig ${ }^{1}$, Sergey Zherebtsov ${ }^{2,3}$, Robert Irsig ${ }^{1}$, Mathias Arbeiter ${ }^{1}$, Christian Peltz ${ }^{1}$, Sebastian Göde ${ }^{1}$ Slawomir Skruszewicz' ${ }^{1}$ Karl-Heinz Meiwes-Broer ${ }^{1}$, Josef Tiggesbäumker ${ }^{1}$, Matthias F. Kling ${ }^{2,3}$ \& Thomas Fennel (iD 1,4

Correction to: Nature Communications https://doi.org/10.1038/s41467-017-01286-w, published online 30 October 2017

The original PDF version of this Article contained an error in Equation 1. The original HTML version of this Article contained errors in Equation 2 and Equation 4. These errors have now been corrected in both the PDF and the HTML versions of the Article.

The original PDF version of this Article contained an error in Equation 1. A dot over the first occurrence of the variable $\mathbf{r}_{i}$ was missing, and incorrectly read:

$$
E_{\mathrm{sp}}=\frac{m}{2}\left|\mathbf{r}_{i}\right|^{2}+V\left(\mathbf{r}_{i}(t), t\right)
$$

The correct form of Equation 1 is as follows:

$$
E_{\mathrm{sp}}=\frac{m}{2}\left|\dot{\mathbf{r}}_{i}\right|^{2}+V\left(\mathbf{r}_{i}(t), t\right)
$$

This has now been corrected in the PDF version of the Article. The HTML version was correct from the time of publication.

The original HTML version of this Article contained errors in Equation 2 and Equation 4.

In Equation 2, a circle over the first occurrence of the variable $\mathbf{r}_{i}$ replaced the intended dot, and incorrectly read:

$$
\dot{E}_{\mathrm{sp}}=q_{i} \mathbf{r}_{i}(t) \cdot \mathcal{E}_{\text {las }}+\frac{\partial}{\partial t} V\left(\mathbf{r}_{i}(t), t\right)
$$

The correct form of Equation 2 is as follows:

$$
\dot{E}_{\mathrm{sp}}=q_{i} \dot{\mathbf{r}}_{i}(t) \cdot \mathcal{E}_{\text {las }}+\frac{\partial}{\partial t} V\left(\mathbf{r}_{i}(t), t\right)
$$

\footnotetext{
${ }^{1}$ Institut für Physik, Universität Rostock, Albert-Einstein-Str. 23, D-18059 Rostock, Germany. ${ }^{2}$ Physik Department, Ludwig-Maximilians-Universität München, Am Coulombwall 1, D-85749 Garching, Germany. ${ }^{3}$ Max-Planck Institut für Quantenoptik, Hans-Kopfermann-Straße 1, D-85748 Garching, Germany. ${ }^{4}$ MaxBorn-Institut für Nichtlineare Optik und Kurzzeitspektroskopie, Max-Born-Straße 2A, D-12489 Berlin, Germany Correspondence and requests for materials should be addressed to J.Täu. (email: josef.tiggesbaeumker@uni-rostock.de) or to M.F.K. (email: matthias.kling@lmu.de) or to T.F. (email: thomas.fennel@uni-rostock.de)
} 
In Equation 4, circles over the first and fifth occurrences of the variable $\mathbf{r}_{i}$ replaced the intended dots, and incorrectly read:

$$
\frac{\mathrm{d}}{\mathrm{d} t} E_{\mathrm{sp}}=m_{i} \mathbf{r}_{i} \cdot \ddot{\mathbf{r}}_{i}+\left[\nabla_{\mathbf{r}_{i}} V\left(\mathbf{r}_{i}, t\right)\right] . \stackrel{\mathbf{r}}{i}_{i}+\frac{\partial}{\partial t} V\left(\mathbf{r}_{i}, t\right)
$$

The correct form of Equation 4 is as follows:

$$
\frac{\mathrm{d}}{\mathrm{d} t} E_{\mathrm{sp}}=m_{i} \dot{\mathbf{r}}_{i} \cdot \ddot{\mathbf{r}}_{i}+\left[\nabla_{\mathbf{r}_{i}} V\left(\mathbf{r}_{i}, t\right)\right] . \dot{\mathbf{r}}_{i}+\frac{\partial}{\partial t} V\left(\mathbf{r}_{i}, t\right)
$$

This has now been corrected in the HTML version of the Article. The PDF version was correct from the time of publication.

Published online: 07 February 2018

(c) Open Access This article is licensed under a Creative Commons Attribution 4.0 International License, which permits use, sharing, adaptation, distribution and Ceproduction in any medium or format, as long as you give appropriate credit to the original author(s) and the source, provide a link to the Creative Commons license, and indicate if changes were made. The images or other third party material in this article are included in the article's Creative Commons license, unless indicated otherwise in a credit line to the material. If material is not included in the article's Creative Commons license and your intended use is not permitted by statutory regulation or exceeds the permitted use, you will need to obtain permission directly from the copyright holder. To view a copy of this license, visit http://creativecommons.org/licenses/by/4.0/.

(c) The Author(s) 2018 